ON THE SUMMABILITY OF DERIVED CONJUGATE SERIES OF THE FOURIER-LEBESGUE TYPE*

BY A. H. SMITH

1. Introduction. We assume throughout that the function $f(x)$ is integrable in the sense of Lebesgue and satisfies the periodicity condition $f(x+2 \pi)=f(x)$; then the series

$$
\sum_{\nu=1}^{\infty}(-1)^{r / 2}\left[\nu^{r}\left(a_{\nu} \sin \nu x-b_{\nu} \cos \nu x\right)\right], \quad(r \text { even }),
$$

and

$$
\sum_{\nu=1}^{\infty}(-1)^{(r-1) / 2}\left[\nu^{r}\left(a_{\nu} \cos \nu x+b_{\nu} \sin \nu x\right)\right], \quad(r \text { odd }),
$$

where $a_{\nu}, b_{\nu}$ are the Fourier coefficients, are defined to be the $r$ th derived conjugate series of the Fourier-Lebesgue type.

In a paper published in 1931†, Bosanquet and Linfoot introduced a regular method of summation which is weaker than that of the Cesàro means of any order $\alpha>0$ and is defined as follows. The series $\sum a_{\nu}$ is said to be summable $(\alpha, \beta)$ to $S$, where either $\alpha>0$, or $\alpha=0, \beta \geqq 0$, if

$$
\sum_{\nu<n}\left[B(1-\nu / n)^{\alpha} \log ^{-\beta}\left(\frac{C}{1-\nu / n}\right) a_{\nu}\right] \rightarrow S, \text { as } n \rightarrow \infty,
$$

for $C$ sufficiently large, $\ddagger$ where $B=(\log C)^{\beta}$.

The object of this paper is to apply the Bosanquet-Linfoot method of summation to the series (1) and (2).§

* Presented to the Society, October 28, 1933.

$\dagger$ L. S. Bosanquet and E. H. Linfoot, On the zero order summability of Fourier series, Journal of the London Mathematical Society, vol. 6 (1931), pp. 117-126.

$\ddagger$ They have shown that it is equivalent to say "for every $C>1$ "; see L. S. Bosanquet and E. H. Linfoot, Generalized means and the summability of Fourier series, Quarterly Journal of Mathematics, Oxford series, vol. 2 (1931), pp. 207229.

$\S$ This method has been applied to Fourier series, the conjugate series and the $r$ th derived Fourier series. See the two papers of Bosanquet and Linfoot given above and A. H. Smith, On the summability of derived series of the FourierLebesgue type, Quarterly Journal of Mathematics, Oxford series, vol. 4 (1933), pp. 93-106. 
2. Notation and Definitions. We define the following functions:

$$
\begin{array}{rlr}
\phi(t) & \equiv f(x+t)+f(x-t)-2 f(x), & \\
\psi(t) & \equiv f(x+t)-f(x-t), & \\
\omega_{r}(t) & \equiv\left\{\begin{array}{lr}
\psi(t), & (r \text { even }), \\
\phi(t), & (r \text { odd }),
\end{array}\right. \\
\Omega_{r}(t) & =\int_{0}^{t}\left|\omega_{r}(u)\right| d u, \\
\theta_{r}(t) & \equiv \int_{0}^{t} \frac{\left|\omega_{r}(u)\right|}{u^{r}} d u, \\
H_{k, \alpha, \beta}(1-u) & \equiv B u^{k}(1-u)^{\alpha-1} \log ^{-\beta}\left(\frac{C}{1-u}\right),
\end{array}
$$

where $B=(\log C)^{\beta}$, for $k \geqq 0, \alpha \geqq 1, \beta \geqq 0$,

$$
\begin{aligned}
Q_{k, \alpha, \beta}(t) & \equiv \int_{0}^{1} H_{k, \alpha, \beta}(1-u) \cos t u d u, \\
\bar{Q}_{k, \alpha, \beta}(t) & \equiv \int_{0}^{1} H_{k, \alpha, \beta}(1-u) \sin t u d u, \\
\bar{\lambda}_{\alpha, \beta}(n, t) & \equiv \frac{1}{\pi} \int_{0}^{n} H_{0, \alpha, \beta}(1-\nu / n) \sin \nu t d \nu, \\
g^{(r)}(x) & \equiv \lim _{\eta \rightarrow 0}-\frac{r !}{\pi} \int_{\eta}^{\infty} \frac{\omega_{r}(t)}{t^{r+1}} d t,
\end{aligned}
$$

whenever the limit on the right hand side exists. The expression $g^{(r)}(x)$, by definition the $r$ th derived conjugate function, is a generalization of the conjugate function

$$
g(x) \equiv g^{(0)}(x) \equiv \lim _{\eta \rightarrow 0}-\frac{1}{\pi} \int_{\eta}^{\infty} \frac{\psi(t)}{t} d t .
$$

The $k$ th derivative of $\bar{\lambda}_{\alpha, \beta}(n, t)$ with respect to $t$ will be denoted by $\bar{\lambda}_{\alpha, \beta}^{(k)}(n, t)$. It can be expressed in terms of $\bar{Q}_{k, \alpha, \beta}(n t)$ or $Q_{k, \alpha, \beta}(n t)$ according as $k$ is even or odd. Finally, we shall define

$$
J \equiv J_{n, r, \beta}(f, x) \equiv(-1)^{r+1} \int_{0}^{\infty} \omega_{r}(t) \bar{\lambda}_{r+1, \beta}^{-(r)}(n, t) d t .
$$


3. Lemmas. The first three lemmas are stated without proof.*

LEMma 1. For $k \geqq 0, \alpha \geqq 1, \beta \geqq 0$, the functions $Q_{k, \alpha, \beta}(t)$ and $\bar{Q}_{k, \alpha, \beta}(t)$ are bounded in $(0, \infty)$, and for large values of $t, c_{k+2}$ being a constant,

$$
\begin{aligned}
Q_{k, \alpha, \beta}(t)+i \bar{Q}_{k, \alpha, \beta}(t)= & \frac{i^{k+1} k !}{t^{k+1}}+\frac{i^{k+2} c_{k+2}}{t^{k+2}} \\
& +O\left(\frac{1}{t^{k+3}}\right)+O\left(\frac{1}{t^{\alpha} \log ^{\beta} t}\right) .
\end{aligned}
$$

Lemma 2. The function $\bar{\lambda}_{\alpha, \beta}^{(k)}(n, t)$ is bounded in $(0, \infty)$ for fixed $n$, where $k \geqq 0, \alpha \geqq 1, \beta \geqq 0$; and for large values of $t$, when $k \geqq 0$ and $\beta \geqq 0$,

$$
\begin{aligned}
\bar{\lambda}_{\alpha, \beta}^{(k)}(n, t)= & \frac{(-1)^{k} k !}{\pi t^{k+1}}+O\left(n^{-\delta} t^{-(k+1+\delta)} \log ^{-\beta} n t\right), \\
\quad(\alpha=k+1+\delta, 0 \leqq \delta<2), & \\
= & \frac{(-1)^{k} k !}{\pi t^{k+1}}+O\left(n^{-2} t^{-(k+3)}\right), \quad(\alpha \geqq k+3) .
\end{aligned}
$$

LEMMA 3. When $\alpha>1, \beta \geqq 0, r \geqq 0$,

$2 \int_{0}^{\infty} \bar{\lambda}_{\alpha, \beta}^{(2 r)}(n, t) \sin \nu t d t$

$$
\begin{array}{lr}
=(-1)^{r} B(1-\nu / n)^{\alpha-1} \log ^{-\beta}\left(\frac{C}{1-\nu / n}\right) \nu^{2 r}, & (0 \leqq \nu \leqq n), \\
=0, & (\nu>n) ;
\end{array}
$$

and

$2 \int_{0}^{\infty} \bar{\lambda}_{\alpha, \beta}^{(2 r+1)}(n, t) \cos \nu t d t$

$$
\begin{aligned}
& =(-1)^{r} B(1-\nu / n)^{\alpha-1} \log ^{-\beta}\left(\frac{C}{1-\nu / n}\right) \nu^{2 r+1}, \quad(0 \leqq \nu \leqq n), \\
& =0, \quad(\nu>n) .
\end{aligned}
$$

* Lemma 1 was proved in an earlier paper, cited above (Lemma 2.1). The proofs of Lemmas 2 and 3 are analogous to those of Lemmas 2.2 and 2.3 of that paper. 
LEMma 4. At every point where $f(x)$ is finite the expression $J$ of (13) is the $[n] t^{*}$ mean of order $(r, \beta \geqq 0)$ of the rth derived conjugate series, $r \geqq 1$, of the Fourier series corresponding to $f(x)$.

Proof. It follows from Lemma 2 that, for fixed $n, \bar{\lambda}_{\alpha, \beta}^{(k)}(n, t)$ is absolutely integrable and of bounded variation in $(0, \infty)$ when

$$
k \geqq 1, \quad \alpha=k+1, \quad \beta \geqq 0 .
$$

Thus, substituting for $[f(x+t)-f(x-t)]$ its Fourier series, employing a theorem of W. H. Young $\dagger$ and Lemma 3 , we have

$$
\begin{aligned}
& \int_{0}^{\infty}[f(x+t)-f(x-t)] \bar{\lambda}_{2 r+1, \beta}^{(2 r)}(n, t) d t \\
&=(-1)^{r} \sum_{\nu<n}\left[B(1-\nu / n)^{2 r} \log ^{-\beta}\left(\frac{C}{1-\nu / n}\right) \nu^{2 r}\right. \\
&\left.\times\left(-a_{\nu} \sin \nu x+b_{\nu} \cos \nu x\right)\right],
\end{aligned}
$$

where $r \geqq 1, \beta \geqq 0$. Similarly, using the fact that

$$
\int_{0}^{\infty} \bar{\lambda}_{2 r+2, \beta}^{(2 r+1)}(n, t) d t
$$

vanishes (integration and Lemma 2), we have at every point where $f(x)$ is finite

$$
\begin{aligned}
\int_{0}^{\infty}[f(x+t)+f(x-t)-2 f(x)] \bar{\lambda}_{2 r+2, \beta}^{(2 r+1)}(n, t) d t \\
=(-1)^{r} \sum_{\nu<n}\left[B(1-\nu / n)^{2 r+1} \log ^{-\beta}\left(\frac{C}{1-\nu / n}\right) \nu^{2 r+1}\right. \\
\left.\times\left(a_{\nu} \cos \nu x+b_{\nu} \sin \nu x\right)\right]
\end{aligned}
$$

where $r \geqq 0, \beta \geqq 0$. By combining (14) and (15), the lemma follows.

4. Summability Theorems. Our main result will now be demonstrated.

* Where $[n]$ is the largest integer not greater than $n$.

$\dagger$ E. W. Hobson, The Theory of Functions of a Real Variable and the Theory of Fourier Series, vol. 2, 2d ed., 1926, p. 583. 
THEOREM 1. If the function $f(x)$ is integrable in the sense of Lebesgue and satisfies the periodicity condition $f(x+2 \pi)=f(x)$, then the rth derived conjugate series, $r \geqq 1$, is summable $(\alpha, \beta)$ for $\alpha=r, \beta>1$ to $g^{(r)}(x)$ whenever the following conditions are satisfied:

(i) $f(x)$ is finite;

(ii) $\Omega_{r}(t)=o\left(t^{r+1}\right)$;

and

(iii) $g^{(r)}(x)$ exists.

(See (5) and (11).)

Proof. Assume that at the point $x$ the conditions of the theorem are satisfied. Let $K_{1}, K_{2}, K_{3}$ denote positive numerical constants. Choose $\epsilon$ arbitrarily small, then $A$ so that

$$
\frac{1}{t^{r+1}} \int_{0}^{t}\left|\omega_{r}(u)\right| d u \leqq \epsilon, \quad(0 \leqq t \leqq A) .
$$

Next choose $n$ so that $n A>e$, divide the interval $(0, \infty)$ into $(0, e / n),(e / n, A)$, and $(A, \infty)$, and denote by $J_{1}, J_{2}$, and $J_{3}$ the respective portions of $J$ of (13).

Expressing $\bar{\lambda}_{r+1, \beta}^{(r)}(n, t)$ in terms of the bounded quantity

$$
\bar{Q}_{r, r+1, \beta}^{(r)}(n t)\left[Q_{r, r+1, \beta}^{(r)}(n t)\right]
$$

if $r$ is even [odd], and then using condition (ii), we have

$$
\left|J_{1}\right| \leqq K_{1}\left(\frac{n}{e}\right)^{r+1} \int_{0}^{e / n}\left|\omega_{r}(t)\right| d t=o(1)
$$

as $n \rightarrow \infty$. In the interval $(e / n, \infty)$, we have

$$
\bar{\lambda}_{r+1, \beta}^{(r)}(n, t)=\frac{(-1)^{r} r !}{\pi t^{r+1}}+O\left(t^{-(r+1)} \log ^{-\beta} n t\right) .
$$

Set $J_{2} \equiv J_{2}^{\prime}+J_{2}^{\prime \prime}$ and $J_{3} \equiv J_{3}^{\prime}+J_{3}^{\prime \prime}$, where

$$
\begin{aligned}
J_{2}^{\prime} & \equiv \frac{-r !}{\pi} \int_{e / n}^{A} \frac{\omega_{r}(t)}{t^{r+1}} d t, \\
\left|J_{2}^{\prime \prime}\right| & \equiv O\left\{\int_{e / n}^{A}\left|\omega_{r}(t)\right| t^{-(r+1)} \log ^{-\beta} n t d t\right\},
\end{aligned}
$$

with similar definitions for $J_{3}^{\prime}$ and $J_{3}{ }^{\prime \prime}$. 
Let us next investigate $J_{2}^{\prime \prime}$. Integrate by parts; the integrated term vanishes for $\beta>1$ as $n \rightarrow \infty$; hence the discussion of $J_{2}{ }^{\prime \prime}$ reduces to that of the two integrals in the expression

$$
\begin{aligned}
& K_{2}(r+1) \int_{e / n}^{A} \Omega_{r}(t) t^{-(r+2)} \log ^{-\beta} n t d t \\
& \quad+K_{2} \beta \int_{e / n}^{A} \Omega_{r}(t) t^{-(r+2)} \log ^{-(\beta+1)} n t d t .
\end{aligned}
$$

Now by (16)

$$
\begin{aligned}
\int_{e / n}^{A} \Omega_{r}(t) t^{-(r+2)} \log ^{-\beta} n t d t & \leqq \epsilon \int_{e / n}^{A} t^{-1} \log ^{-\beta} n t d t \\
& \leqq \frac{\epsilon}{\beta-1}\left(1-\log ^{-\beta+1} n A\right),
\end{aligned}
$$

which for $\beta>1$ is arbitrarily small with $\epsilon$, since $n A>e$. Similarly, for $\beta>1$, the second integral of (19) is arbitrarily small with $\epsilon$. Thus for $\beta>1$,

$$
J_{2}^{\prime \prime} \rightarrow 0,
$$

as $n \rightarrow \infty$. Finally, since $f(x)$ is periodic, choose $q$ so that $2(q-1) \pi \leqq A<2 q \pi ;$ then

and thus for $\beta>1$,

$$
\begin{array}{r}
\left|J_{3}^{\prime \prime}\right| \leqq K_{3}\left[\int _ { 0 } ^ { 2 \pi } | \omega _ { r } ( t ) | d t \left\{\frac{1}{A^{r+1} \log ^{\beta} n A}\right.\right. \\
\left.\left.+\frac{1}{(2 \pi)^{r+1}} \sum_{\nu=q}^{\infty} \frac{1}{\nu^{r+1} \log ^{\beta} 2 n \pi \nu}\right\}\right],
\end{array}
$$

$$
J_{3}{ }^{\prime \prime} \rightarrow 0,
$$

as $n \rightarrow \infty$. Hence from (17), (18), (19), (20), and (21), we have

$$
\lim _{n \rightarrow \infty} J=\lim _{n \rightarrow \infty} \frac{-r !}{\pi} \int_{e / n}^{\infty} \frac{\omega_{r}(t)}{t^{r+1}} d t=\lim _{\eta \rightarrow 0} \frac{-r !}{\pi} \int_{\eta}^{\infty} \frac{\omega_{r}(t)}{t^{r+1}} d t,
$$

provided the latter limit exists. Thus the theorem is demonstrated.

THEOREM 2. The condition

$$
\Omega_{r}(t)=o\left(t^{r+1}\right)
$$

is equivalent to the condition $\theta_{r}(t)=o(t)$. (See (6).) 
Proof. We have

$$
\left.\theta_{r}(t)=\Omega_{r}(s) s^{-r}\right]_{0}^{t_{r}}+r \int_{0}^{t} \Omega_{r}(s) s^{-(r+1)} d s=o(t)
$$

at every point for which

$$
\Omega_{r}(s)=o\left(s^{r+1}\right) .
$$

We have also

$$
\begin{aligned}
\Omega_{r}(t)= & \left.\int_{0}^{t} \frac{\left|\omega_{r}(s)\right|}{s^{r}} s^{r} d s=s^{r} \theta_{r}(s)\right]_{0}^{t} \\
& -r \int_{0}^{t} \theta_{r}(s) s^{r-1} d s=o\left(t^{r+1}\right)
\end{aligned}
$$

at every point for which $\theta_{r}(s)=o(s)$. Hence the theorem is proved.

5. Case $r=0$. We note that if $r=0$, it can be stated that the conjugate Fourier series is summable $(0, \beta>1)$ almost everywhere to $g(x)$ of $(12)$.

This follows since for $\beta>1, \bar{\lambda}_{1, \beta}(n, t)$, for fixed $n$, is of bounded variation over $(0, \infty)$ and tends to zero as $t \rightarrow \infty$, and since for $\alpha=1, \beta>0, r=0$, Lemma 3 is satisfied. Hence, employing as before Young's theorem, we find that Lemma 4 is valid for $r=0$. Moreover, in this case the calculations of Theorem 1 hold and its conditions are satisfied almost everywhere.*

\section{BROWN UNIVERSITY}

* That $g(x)$ exists almost everywhere was proved by A. Plessner, Zur Theorie der konjugierten trigonometrischen Reihen, Mitteilungen des Mathematischen Seminars der Universität Giessen, Heft 10 (1923). 